\title{
AUTORÍA EN ARTÍCULOS BIOMÉDICOS: EL CASO DE IMAGINOLOGÍA
}

\section{Dr. Claudio Silva Fuente-Alba}

Médico Radiólogo, Departamento de Imágenes, Clínica Alemana - Facultad de Medicina Universidad del Desarrollo. Santiago, Chile.

Miembro Comité Editorial Revista Chilena de Radiología.

\section{AUTHORSHIP IN BIOMEDICAL ARTICLES. THE CASE OF IMAGING}

\begin{abstract}
The definition of authorship is a subject of ongoing discussion wherein elements of prestige and academic career are involved. As a potential source of conflicts, it must be handled with extreme strictness to avoid difficulties when presenting scientific outcomes. Both a review of international standards relating to regarding authorship, accepted to date, and the position of the editorial board of the Revista Chilena de Radiología are presented.

Keywords: Authorship coercion, International Committee of Medical Journal Editors, Gift authorship, Ghost authorship, Uniform requirements for manuscripts submitted to biomedical journals.
\end{abstract}

Resumen: La definición de autoría es un tema de discusión permanente, donde se ven involucrados elementos de prestigio y carrera académica. Siendo una potencial fuente de conflictos, debe ser manejado con extrema rigurosidad, para evitar dificultades al momento de presentar resultados científicos. Se presenta una revisión de los criterios internacionales referidos a autoría, aceptados a la fecha, y la postura del cuerpo editorial de la Revista Chilena de Radiología.

Palabras clave: Autoría por obsequio, Autoría fantasma, Autoría por coerción, Comité Internacional de Editores de Revistas Médicas, Requisitos de uniformidad para manuscritos enviados a revistas biomédicas.

\section{Introducción}

La autoría en los artículos biomédicos siempre ha sido asociada a la actividad académica, al deseo de aportar conocimiento hacia la comunidad médi-

Silva C y cols. Autoría en artículos biomédicos: El caso de imaginología. Rev Chil de Radiol 2010; 16(1): 17-23.

Correspondencia: Dr. Claudio Silva F. drsilvafa@gmail.com

Trabajo recibido el 08 de octubre de 2009, aceptado para publicación el 26 de enero de 2010. ca y fomentar el progreso del quehacer actual en medicina. La imaginología no ha sido la excepción y en la actualidad existen múltiples revistas del área, algunas de ellas enfocadas a subespecialidades, cuyo norte no es otro sino ser una vía de extensión de los hallazgos en distintas esferas.

Con el correr de los años, las publicaciones se transformaron en un parámetro medible de la "producción" intelectual o académica de un médico y éste se convirtió en uno de los elementos que determinan el ascenso en la carrera académica. Ello llevó a una distorsión del proceso intelectual, donde se creó la necesidad de presentar autoría de un determinado número de artículos que permitiesen continuar mejorando su calificación, por ende apareciendo como "autor" o "co-autor" en múltiples publicaciones.

En 1942, Logan Wilson, un sociólogo académico de la Universidad de Texas, acuñó por primera vez el término "publish or perish"(1) y en la década de los sesenta se hizo extensivo a la medicina, siendo interpretado en su inicio como un motivo noble de tomar un tiempo para redactar el conocimiento adquirido para ser compartido. Ello determinó, en décadas posteriores, un incremento en el número de artículos publicados, destacando también el aumento aún mayor de autores involucrados (Gráfico 1). En radiología, esto se vio refrendado en el estudio de Chew et $\mathrm{al}^{(2)}$ en el año 1988, que analizó las tendencias en las dos revistas de mayor difusión de la especialidad (Radiology y AJR). Las exigencias de variadas instituciones a aumentar el número de publicaciones, ha generado una serie de problemas éticos en relación a autoría, apareciendo casos lamentables de fraude científico que han afectado todas las áreas biomédicas, donde la radiología no ha sido la excepción.

\section{Fraude en Radiología: El caso Slutsky}

Robert Slutsky era un médico de la Universidad de California San Francisco (UCSF), con especialidad en cardiología, que se embarcó en la beca de radiología. Durante el curso de estas becas de especialidad se 


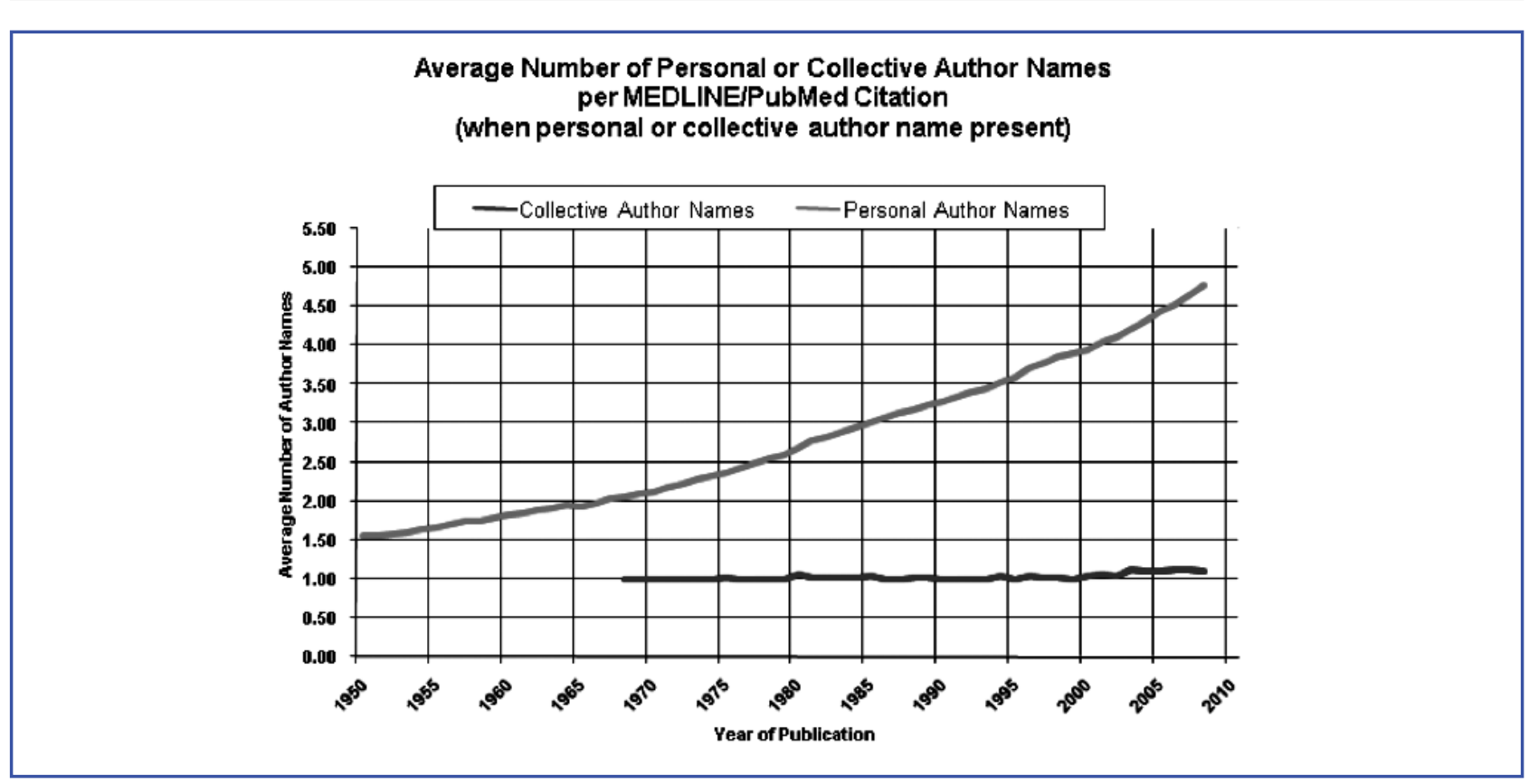

Gráfico 1. Tendencia del número de autores en artículos registrados en Medline.

Disponible en: http://www.nlm.nih.gov/bsd/authors1.html • Acceso 5 de Octubre, 2009

inició en el campo de las publicaciones científicas, siendo especialmente prolífico en esta área. Se ha determinado que, en su época de becado publicó 137 manuscritos con 93 distintos autores ${ }^{(3)}$, en un período de 7 años, estimándose que en ese período completaba un artículo cada 13 días ${ }^{(4)}$. Sin embargo, una serie de inconsistencias al abordar sus fuentes de información y datos, precipitaron una denuncia de sus compañeros de facultad, que llevó a la creación de una Comisión Investigadora por parte de la UCSF.

En el curso de esta investigación, se pudo comprobar que de los 137 artículos publicados, 77 calificaban como "válidos", 48 como "dudosos" y 12 fueron considerados fraudes ${ }^{(4)}$. Dentro de estos fraudes, hubo experimentos que nunca se realizaron, procedimientos incorrectos, mediciones no realizadas y reporte de análisis estadísticos inexistentes ${ }^{(5)}$. A raíz de ésto, se ordenó 17 retracciones a ser informadas a revistas tan prestigiosas como Radiology, Critical Care Medicine y Journal of the American College of Cardiology, entre otros.

Fruto de esta investigación, el Dr. Slutsky fue sancionado, siendo expulsado del laboratorio de investigación y a continuación renunció a su residencia en Radiología en la UCSF. Como efecto de esta revisión, también fueron sancionados muchos de los co-autores, dado que no cumplieron con la prolijidad de certificar que el o los trabajos donde aparecen como firmantes fueran reales y/o consistentes. Algunos de ellos incluso fueron despojados de sus cargos académicos.

\section{¿Qué es autoría?}

La definición de autoría no ha sido fácil ni libre de polémicas. Un primer intento de estandarización de lo que se considera como autor surge a raíz de la creación del Grupo de Vancouver en 1978. Este trabajo involucró a editores de las revistas biomédicas de mayor prestigio y, en 1997 se constituyó como la ICMJE (International Committee of Medical Journal Editors), tras lo cual han emitido normas formales cada dos años, con su última actualización publicada en Octubre de $2007^{(6)}$.

La ICMJE hace una distinción entre los autores (authorship), en base a sus contribuciones (contributorship). Se considera autor a aquel que ha hecho aportes intelectuales substantivos y significativos a un trabajo. Ello se basa en tres puntos:

a) Contribuciones sustanciales en el diseño, adquisición de datos, o análisis e interpretación.

b) Redacción del borrador del trabajo o revisión crítica de él para agregar contenido intelectualmente importante.

c) Aprobación final de la versión a publicar (Tabla I).

Se exige que los autores deban cumplir con las tres condiciones, de forma de poder tomar pública responsabilidad del contenido y cada sección de él. Todos aquellos que no cumplan con los criterios de autoría deben ser mencionados en sección de agradecimientos $^{(6)}$. Ejemplo de esto último serían aquellos que ayudaron en la obtención de fondos, médicos clínicos que aportaron pacientes o jefes de servicios que sólo proporcionaron apoyo general (uso de equipos, por ejemplo).

La tendencia internacional es a transparentar el rol de cada autor referido a estos tres criterios por lo que algunas revistas ya exigen explicitar el aporte, 
Tabla I. Pauta de Autoría del International Committee of Medical Journal Editors (ICMJE).

Todos los criterios presentados a continuación deben cumplirse para calificar como autor:

1. Contribución importante y sustancial en la concepción y diseño o adquisición de datos o, análisis e interpretación de los datos.

2. Redactar o revisar críticamente el artículo generado siendo un aporte intelectual a éste.

3. Aprobación final de la versión a ser publicada.

como: BMJ, Annals of Internal Medicine, Lancet, Radiology y, en nuestro medio, la Revista Médica de Chile. En algunos casos, se solicita además enumerar los aportes realizados por cada autor y se pide que uno de ellos asegure que toda la ejecución ha sido llevada a cabo en forma rigurosa, constituyéndose en garante de aquello.

\section{¿Por qué debiera importar la autoría?}

El proceso de generar nuevo conocimiento es un trabajo arduo, que implica muchas horas de trabajo intelectual para poder afinar y definir una pregunta de investigación válida, crear un diseño metodológicamente apto para contestar la inquietud y determinar la logística requerida para su ejecución. Involucra también una dedicación particular en reclutar pacientes, recabar datos, procesarlos, interpretarlos y finalmente plasmar todo éso en un artículo destinado para publicación o difusión dentro del medio. Por ello, determinar quién merece ser incluido como autor, no sólo se refiere a quién debe recibir el crédito por el trabajo realizado, sino también a quién puede hacerse responsable por la información que se obtuvo.

Esto se traduce en situaciones extremadamente complejas, donde se generan categorías indebidas de autoría ${ }^{(7-9)}$, entre las que cabe mencionar:

"Autoría por obsequio": definido como un reconocimiento en el contexto de agradecimiento o, por una dependencia jerárquica. Ejemplo de ello sería incluir a un mentor o a una eminencia de un tema, con la esperanza de aumentar las posibilidades de publicación o, incluir a un Jefe de Departamento que no ha tenido participación alguna en la investigación (en ambientes académicos, generalmente referido a residentes y sus jefes de carrera).

"Autoría fantasma": se refiere a publicaciones donde no son incluidos en la publicación final autores que contribuyeron significativamente a un trabajo. A veces esto se asocia a proyectos a gran escala, donde aquellos encargados de la ejecución y análisis de datos no son reconocidos en el trabajo final.

"Autoría por coerción": en este caso, una autoridad ejerce presión ilegítima sobre los autores para ser incluido, con manifiesta o velada intención de perjudicar a los investigadores de no ser acogida su solicitud.

En lo expuesto hay elementos que limitan con la ética profesional, que son más frecuentes de lo que uno se imagina.

\section{La evidencia en Radiología}

El número de autores involucrados en un artículo debiera ser proporcional a la complejidad del tema tratado y representar a aquellos que tuvieron una participación destacada dentro del proceso intelectual de desarrollo y ejecución del proyecto. Sin embargo, numerosas publicaciones han identificado tendencias peculiares, que impresionan alejarse de esta premisa, donde el número de autores crece en forma manifiesta. Ello ha generado incluso el caso de un artículo con 2.516 autores involucrados ${ }^{(10)}$; en Medline, el mayor número de autores registrados es de 976 , agrupado en "los investigadores GUSTO"(11).

En radiología esta tendencia ya se identifica en 1988, cuando Felix Chew ${ }^{(2)}$ realizó una revisión de los artículos publicados en AJR el año 1982 y posteriormente revisó un período de cinco años, para observar el cruce con otros artículos de estos autores. Se pudo ver que la mediana de número de coautoría estaba entre 5 y 6 artículos, identificándose incluso un caso con 82 coautorías en este período (Gráfico 2 y Tabla II). Ante esta situación, es válido preguntarse si es esperable que un autor se repita en tantos trabajos; si su contribución fuese importante, eso refleja una dedicación al tema académico y sin duda debiera ser así.

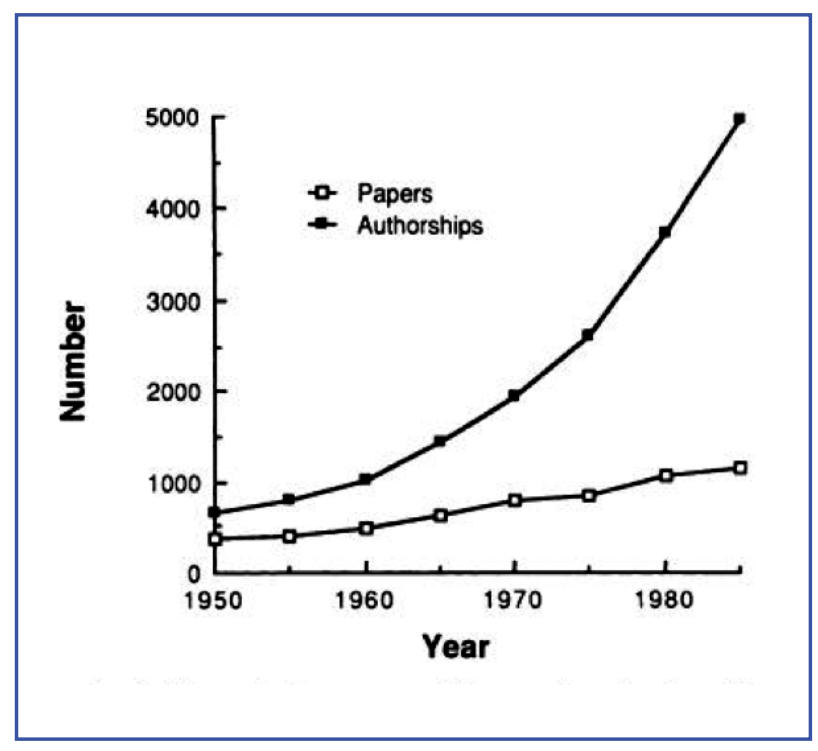

Gráfico 2. Muestra un incremento exponencial del número de autores, con una progresión lineal del número de artículos publicados en AJR y Radiology. 
Tabla II. Distribución del número de coautores acumulados por 136 radiólogos-investigadores, 1980-1985.

\begin{tabular}{|c|c|c|c|}
\hline $\begin{array}{c}\text { № de } \\
\text { Coautorías }\end{array}$ & $\begin{array}{c}\mathrm{N} \text { o de } \\
\text { Investigadores }\end{array}$ & $\begin{array}{l}\text { Porcentaje ac } \\
\text { de } \\
\text { Investigadores }\end{array}$ & $\begin{array}{l}\text { umulado } \\
\text { de } \\
\text { Coautorías }\end{array}$ \\
\hline 0 & 4 & 2.9 & 0.0 \\
\hline 1 & 32 & 26.5 & 2.0 \\
\hline 2 & 14 & 36.8 & 3.7 \\
\hline 3 & 3 & 39.0 & 4.2 \\
\hline 4 & 7 & 44.1 & 6.0 \\
\hline 5 & 7 & 49.3 & 8.1 \\
\hline 6 & 6 & 53.7 & 10.3 \\
\hline 7 & 1 & 54.4 & 10.8 \\
\hline 8 & 9 & 61.0 & 15.2 \\
\hline 9 & 2 & 62.5 & 16.3 \\
\hline 10 & 2 & 64.0 & 17.5 \\
\hline 11 & 1 & 64.7 & 18.2 \\
\hline 12 & 4 & 67.6 & 21.2 \\
\hline 13 & 4 & 70.6 & 24.4 \\
\hline 14 & 2 & 72.1 & 26.1 \\
\hline 15 & 2 & 73.5 & 27.9 \\
\hline 16 & 3 & 75.7 & 30.9 \\
\hline 17 & 5 & 79.4 & 36.1 \\
\hline 20 & 2 & 80.9 & 38.6 \\
\hline 21 & 1 & 81.6 & 39.9 \\
\hline 22 & 1 & 82.4 & 41.2 \\
\hline 23 & 1 & 83.1 & 42.6 \\
\hline 24 & 1 & 83.8 & 44.1 \\
\hline 26 & 2 & 85.3 & 47.3 \\
\hline 27 & 1 & 86.0 & 49.0 \\
\hline 28 & 2 & 87.5 & 52.4 \\
\hline 29 & 1 & 88.2 & 54.2 \\
\hline 30 & 3 & 90.4 & 59.8 \\
\hline 32 & 1 & 91.2 & 61.7 \\
\hline 33 & 1 & 91.9 & 63.8 \\
\hline 37 & 2 & 93.4 & 68.3 \\
\hline 40 & 1 & 94.1 & 70,8 \\
\hline 44 & 1 & 94.9 & 73.5 \\
\hline 48 & 1 & 95.6 & 76.4 \\
\hline 51 & 1 & 96.3 & 79.6 \\
\hline 58 & 1 & 97.1 & 83.1 \\
\hline 60 & 1 & 97.8 & 86.8 \\
\hline 63 & 1 & 98.5 & 90.7 \\
\hline 69 & 1 & 99.3 & 95.0 \\
\hline 82 & 1 & 100.0 & 100.0 \\
\hline \multicolumn{4}{|c|}{$\begin{array}{l}\text { Nota. Un total de } 1625 \text { coautorías concentraron } 136 \text { investiga- } \\
\text { dores. } \\
\text { Reproducido con autorización del autor desde Chew F. Coau- } \\
\text { thorship in Radiology Journals. AJR Am J Roentgenol. 1988; } \\
\text { 150: } 23-26 .\end{array}$} \\
\hline
\end{tabular}

Slone et al, estudiaron la contribución de los autores en una muestra que consideró los artículos publicados en AJR durante los años 1992 y $1993^{(12)}$. De los 437 artículos analizados, se seleccionaron aquellos redactados por autores residentes en EE.UU., estableciendo un grupo muestral de 286. En ellos se evaluó, en forma anónima y vía cuestionario, la contribución en diseño, recolección/análisis de datos y redacción final, tabulando además el cargo académico de cada uno de los autores. En este estudio se pudo determinar que, a contar del cuarto autor $(y$ hasta el décimo evaluado), entre un 31 y $47 \%$ había contribuido a los elementos de diseño, recolección de datos, análisis de datos y un $61 \%$ participó en la preparación del artículo final. Lo más preocupante es que sólo un $31 \%$ contribuyó en tres o cuatro de las categorías que definen autoría.

Casi una década después, en 2003, Hwang et al(13) (Tabla III), hicieron una evaluación similar en Radiology; se observó que pese a estar en vigencia los parámetros requeridos por ICMJE, hubo un bajo cumplimiento en lo que se refiere a la redacción, aportes críticos y aprobación del manuscrito final, que en definitiva será lo que represente todo el trabajo en el cual los autores invirtieron tiempo y recursos. Así también se puede ver que esta tendencia es más marcada mientras más autores participan.

En el medio nacional, también tenemos evaluaciones del tema. En el año 2002, Jacard M et al(14), estudiaron la tendencia en número de autores y calificación de autoría en Revista Médica de Chile entre los años 1969 y 2000 . Se observó que la tendencia muestra un incremento del número de autores en los artículos de investigación, lo que pudiera estar justificado si estuviera dado por más trabajo colaborativo. Sin embargo, al estudiar el cumplimiento de los requisitos ICMJE, se vio que el $52.5 \%$ de los autores de trabajos de investigación y sólo un $37.8 \%$ de los casos clínicos, cumplían en rigor con el calificativo de autor (Tabla IV y V).

\section{¿Cuántos radiólogos se requieren para un trabajo de investigación?}

No hay una forma sencilla de contestar esta pregunta. En los tiempos actuales, donde cada vez se desarrollan áreas más específicas, muchas veces se generan grupos de trabajo en los que participa un número importante de radiólogos en un proyecto de investigación. Por ello, cuando la magnitud de un trabajo, ya sea en diseño, extensión o logística, ha requerido la participación de múltiples actores, es de toda justicia que ello se vea reflejado en la publicación.

Los comités editoriales de las distintas revistas médicas y de especialidad han tenido dificultades para enfrentar este tema, si bien hay algunos puntos donde existe un consenso general. Se entiende que, en publicaciones donde hay un desarrollo metodológico menor, la participación de los autores también debe ser menor. Por ello, en lo que se refiere a cartas al editor y reportes de casos, rara vez se aceptan más de 3 autores. Por otro lado, se espera que haya participación de un mayor número de autores si estamos evaluando un trabajo de cohorte, caso-control 
Tabla III. Cumplimiento de las condiciones de autoría ICMJE.

ICMJE Condiciones*

\begin{tabular}{|c|c|c|c|c|c|}
\hline \multirow[b]{2}{*}{ Característica de artículo } & \\
\hline & Primero & Segundo & Tercero(\%) ${ }^{\dagger}$ & Todo & $p$ Value $\ddagger$ \\
\hline \multicolumn{5}{|c|}{ Posición del autor en referencia } & $<.001$ \\
\hline Primero $(n=1,068)$ & $1,065(99.7)$ & $1,058(99.1)$ & 100 & $1,056(98.9)$ & \\
\hline Segundo $(n=1,068)$ & $1,043(97.7)$ & $934(87.5)$ & 100 & $911(85.3)$ & \\
\hline Último $(n=1,068)$ & $858(80.3)$ & $907(84.9)$ & 100 & $710(66.5)$ & \\
\hline Medio $(n=3,482)$ & $3,149(90.4)$ & $2,160(62.0)$ & 100 & $1,837(52.8)$ & \\
\hline \multicolumn{5}{|l|}{ Nacionalidad autor } & $<.001$ \\
\hline Norteamericano $(n=3,364)$ & $3,187(94.7)$ & $2,809(83.5)$ & 100 & $2,631(78.2)$ & \\
\hline Internacional $(n=3,322)$ & $2,930(88.2)$ & $2,250(67.7)$ & 100 & $1,883(56.7)$ & \\
\hline \multicolumn{5}{|l|}{ Año de publicación } & .18 \\
\hline $1998(n=2,152)$ & $1,985(92.2)$ & $1,586(73.7)$ & 100 & $1,426(66.3)$ & \\
\hline $1999(n=2,176)$ & $2,002(92.0)$ & $1,667(76.6)$ & 100 & $1,499(68.9)$ & \\
\hline $2000(n=2,358)$ & $2,128(90.2)$ & $1,806(76.6)$ & 100 & $1,589(67.4)$ & \\
\hline \multicolumn{5}{|l|}{ № de autores por artículo } & $<.001$ \\
\hline $3(n=312)$ & 299(95.8) & 289(92.6) & 100 & $276(88.5)$ & \\
\hline $4(n=532)$ & $509(95.7)$ & $464(87.2)$ & 100 & $441(82.9)$ & \\
\hline $5(n=925)$ & $852(92.1)$ & $787(85.1)$ & 100 & $717(77.5)$ & \\
\hline $6(n=1,266)$ & 1,156(91.3) & 1,004(79.3) & 100 & 898(70.9) & \\
\hline $7(n=1,029)$ & 939(91.3) & $748(72.7)$ & 100 & $661(64.2)$ & \\
\hline $8(n=1,024)$ & $919(89.7)$ & $697(68.1)$ & 100 & $595(58.1)$ & \\
\hline $9(n=684)$ & 611(89.3) & $456(66.7)$ & 100 & $390(57.0)$ & \\
\hline $10(n=400)$ & $368(92.0)$ & $256(64.0)$ & 100 & $225(56.3)$ & \\
\hline$>10(n=514)$ & 462(89.9) & $358(69.6)$ & 100 & $311(60.5)$ & \\
\hline TOTAL $(\mathrm{n}=6,686)$ & $6,115(91.5)$ & $5,059(75.7)$ & 100 & $4,514(67.5)$ & \\
\hline
\end{tabular}

Nota: Números en paréntesis corresponden a porcentajes.

* Primera condición se refiere a contribuciones en concepción y diseño, adquisición de datos y análisis e interpretación de los datos; la segunda contribución se refiere a aportes en la redacción del artículo y su revisión crítica con aportes de importancia; la tercera condición hace referencia a contribuciones a la versión a ser publicada.

† Se asumió que la tercera condición se cumplió basada en la inclusión y firma del formulario de derecho de autor exigido al momento de remitirse para publicación.

$\ddagger P$ values obtenidos con test $\mathrm{ji}-2$.

(Publicado originalmente en inglés en Su Hwang S, Song HH, Baik JH, et al. Researcher contributions and fulfillment of ICMJE authorship criteria: analysis of autor contribution lists in research article with multiple authors, Radiology 2003; 226:16-23; publicado online en Noviembre 13, 2002, doi:10.1148/radiol.2261011255. Radiology es publicado por la Sociedad Radiológica de Norte América, Inc. (RSNA) @ RSNA. Reproducido con autorización de RSNA.

Originally published in English in Su Hwang S, Song HH, Baik JH, et al. Researcher contributions and fulfillment of ICMJE authorship criteria: analysis of autor contribution lists in research article with multiple authors, Radiology 2003; 226:16-23; published online November 13, 2002, doi:10.1148/radiol.2261011255. Radiology is owned and published be the Radiological Society of North America, Inc. (RSNA) @ RSNA. Reprinted with permission of RSNA.) 
Tabla IV. Número de autores por artículo, por año (promedios +/- DS).

\begin{tabular}{|c|c|c|c|c|c|c|c|c|}
\hline \multirow[b]{2}{*}{ Clase de Artículo } & \multirow[b]{2}{*}{$\mathrm{n}$} & \multicolumn{7}{|c|}{ Años } \\
\hline & & 1969 & 1979 & 1984 & 1989 & 1994 & 1999 & 2000 \\
\hline Artículos de investigación & 661 & $3,9 \pm 1,6$ & $4,5 \pm 1,9$ & $3,8 \pm 1,7$ & $4,9 \pm 2,0$ & $5,7 \pm 2,5^{\star}$ & $5,2 \pm 2,6^{*}$ & $5,4 \pm 2,2^{*}$ \\
\hline Casos clínicos & 201 & $4,8 \pm 3,0$ & $3,5 \pm 1,7$ & $3,6 \pm 1,6$ & $4,3 \pm 1,5$ & $4,4 \pm 2,0$ & $4,1 \pm 1,6$ & $4,4 \pm 2,2$ \\
\hline Otros $^{(1)}$ & 117 & $2,4 \pm 1,1$ & $3,8 \pm 2,0$ & $3,2 \pm 1,7$ & $3,5 \pm 1,7$ & $3,4 \pm 2,1$ & $3,7 \pm 2,1$ & $2,5 \pm 1,4$ \\
\hline TOTAL & 979 & $4,3 \pm 3,5$ & $4,1 \pm 1,9$ & $3,6 \pm 1,7$ & $4,6 \pm 1,8$ & $5,1 \pm 2,5$ & $4,8 \pm 2,4$ & $4,7 \pm 2,3$ \\
\hline
\end{tabular}

Tabla V. Calificación de la autoría en los manuscritos publicados en la Revista Médica de Chile durante un año (2000-2001).

\begin{tabular}{|c|c|c|c|c|c|c|c|c|}
\hline \multirow{3}{*}{ Clase de artículo } & \multirow{2}{*}{\multicolumn{2}{|c|}{$\begin{array}{c}\text { Número } \\
\text { de autores }\end{array}$}} & \multicolumn{4}{|c|}{ Categoría de la autoría } & & \\
\hline & & & \multicolumn{2}{|c|}{ Justificada } & \multicolumn{2}{|c|}{ Parcial } & \multicolumn{2}{|c|}{ Injustificada } \\
\hline & $\mathrm{n}$ & $\%$ & $\mathrm{n}$ & $\%$ & $\mathrm{n}$ & $\%$ & $\mathrm{n}$ & $\%$ \\
\hline Artículos de investigación & 690 & 100 & 362 & 52,5 & 290 & 42,0 & 38 & 5,5 \\
\hline Casos clínicos & 156 & 100 & 59 & 37,8 & 77 & 49,4 & 20 & 12,8 \\
\hline $\operatorname{Otros}^{(2)}$ & 75 & 100 & 51 & 68,0 & 23 & 30,7 & 1 & 1,3 \\
\hline TOTAL & 92 & 100 & 472 & 51,2 & 390 & 42,3 & 59 & 6,4 \\
\hline \multicolumn{9}{|c|}{ (1) Aplicando criterios del ICMJE, versión 2001 (visible en http://www.icmje.org) } \\
\hline
\end{tabular}

o estudio de eficacia de prueba diagnóstica.

Se han propuesto múltiples estrategias para enfrentar este tema, algunas de los cuales están en análisis y discusión para eventualmente ser implementadas. Dentro de ellas se han propuesto categorizaciones de los autores, basados en cuántos criterios ICMJE han sido cumplidos (dicotomizar o tricotomizar), anonimizar las publicaciones o utilizar un listado alfabético; incluso se ha propuesto que entidades externas analicen las contribuciones de cada autor y discriminen, lo que implica un proceso activo por parte de los autores, Comités científicos institucionales y Comités Editoriales.

Por lo pronto, algunas revistas de la especialidad han colocado límites a ciertas contribuciones, como AJR que limita a siete los autores para sus artículos de investigación original, cuatro en comentario/opinión y tres para cartas al editor / cartas ilustradas. A la fecha, Radiology, European Radiology, Pediatric Radiology y Journal of Computed Assisted Tomography, entre otros, aún no han tomado una determinación.
Es por ello que la Revista Chilena de Radiología ha entrado en una fase de análisis profundo en este sentido, donde por lo pronto, se solicitará la declaración de contribución de los autores, basándose en lo reflejado en la Tabla I. A la fecha, se ha hecho una recomendación referida al número de autores según consta en las "Instrucciones a los autores"(15). Dejamos abierta la posibilidad de debate y discusión, invitándolos a enviar sus cartas al Editor, a través de la sección correspondiente.

\section{Bibliografía}

1. Garfield E. What Is The Primordial Reference For The Phrase 'Publish Or Perish'? The Scientist 1996; 10(12): 11-12.

2. Chew F. Coauthorship in Radiology Journals. AJR Am J Roentgenol 1988; 150: 23-26.

3. Sox HC, Rennie D. Research Misconduct, Retraction, and Cleansing the Medical Literature: Lessons from the Poehlman Case. Ann Intern Med 2006; 144: 609613.

4. Whitely WP, Rennie D, Hafner AW. The Scientific 
Community's Response to Evidence of Fraudulent Publication The Robert Slutsky Case. JAMA 1994; 272: 170-173.

5. Engler RL, Covell JW, Friedman PJ, Kitcher PS, Peters RM. Misrepresentation and responsibility in medical research. N Engl J Med 1987; 317(22): 1383-1389.

6. ICMJE. Uniform Requirements for Manuscripts Submitted to Biomedical Journals: Ethical Considerations in the Conduct and Reporting of Research: Authorship and Contributorship. http://www.icmje.org/ethical_1author. html Acceso Octubre 5, 2009.

7. Feeser VR, Simon JR. The ethical assignment of authorship in scientific publications: issues and guidelines. Acad Emerg Med 2008; 15(10): 963-969.

8. Berquist TH. Authorship: Did I Really Contribute? AJR Am J Roentgenol 2009; 193: 915-916.

9. Castillo M. Authorship and Bylines. AJNR Am J Neuroradiol 2009; 30: 1455-1458.

10. ALEPH Collaboration et al. Precision electroweak measurements on the $Z$ resonance. Physics Reports
2006; 427: 257-454.

11. The GUSTO Investigators. An International Randomized Trial Comparing Four Thrombolytic Strategies for Acute Myocardial Infarction. NEJM 1993; 329: 673-682.

12. Slone RM. Coauthors' contributions to major papers published in the AJR: frequency of undeserved coauthorship. AJR Am J Roentgenol 1996; 167(3): 571-579.

13. Hwang S, Song HH, Baik JH, et al. Researcher contributions and fulfillment of ICMJE authorship criteria: analysis of autor contribution lists in research article with multiple authors. Radiology 2003; 226: 16-23.

14. Jacard M, Herskovic V, Hernández I, Reyes H. Un análisis de la autoría en los artículos publicados en la Revista Médica de Chile. Rev Méd Chile 2002; 130: 1391-1398.

15. Instrucciones a los autores. Revista Chilena de Radiología [Internet], Sociedad Chilena de Radiología. 2009 [acceso 14 de Diciembre 2009] Disponible en: http://www.scielo.cl/revistas/rchradiol/einstruc.htm 